\title{
Asymptotic analysis of a new type of multi-bump, self-similar, blowup solutions of the Ginzburg-Landau equation
}

\author{
V. ROTTSCHÄFER \\ Mathematical Institute, Leiden University, P.O. Box 9512, 2300 RA Leiden, the Netherlands \\ email: vivi@math.leidenuniv.nl
}

(Received 21 Febuary 2012; revised 11 September 2012; accepted 13 September 2012;

first published online 29 October 2012)

\begin{abstract}
We study of a new type of multi-bump blowup solutions of the Ginzburg-Landau equation. Multi-bump blowup solutions have previously been found in numeric simulations, asymptotic analysis and were proved to exist via geometric construction. In the geometric construction of the solutions, the existence of two types of multi-bump solutions was shown. One type is exponentially small at $\xi=0$, and the other type of solutions is algebraically small at $\xi=0$. So far, the first type of solutions were studied asymptotically. Here, we analyse the solutions which are algebraically small at $\xi=0$ by using asymptotic methods. This construction is essentially different from the existing one, and ideas are obtained from the geometric construction. Hence, this is a good example of where both asymptotic analysis and geometric methods are needed for the overall picture.
\end{abstract}

Key words: Ginzburg-Landau equation; Multi-bump blowup solutions

\section{Introduction}

The Ginzburg-Landau equation (GL) arises as a model equation in various problems coming from physics, biology and chemistry. For example, in Rayleigh-Bénard convection, Taylor-Couette flow, nonlinear optics, models of turbulence, superconductivity, superfluidity and reaction-diffusion systems, it can be derived, see $[3,6,7,9,15]$ and the review paper [2]. More general, in 'marginally unstable' systems of nonlinear partial differential equations defined on unbounded domains, the leading order behaviour of small perturbations is described by the GL as a normal form, [8]. Since the derivation loses its validity for large amplitude, it is of particular interest to study existence and stability of blowup solutions to the GL.

We study the GL written in the following form

$$
i \frac{\partial \Phi}{\partial t}+(1-i \varepsilon) \Delta \Phi+(1+i b \varepsilon)|\Phi|^{2} \Phi=0
$$

where $\Phi(x, t)$ is a complex amplitude, $x \in \mathbf{R}^{d}, \varepsilon>0, t>0$ and $b$ is an $O(1)$ parameter. Although the parameter $d$ represents the dimension, we also consider non-integer values of $d$, as will be explained in more detail later on. This equation can be obtained by 
rescaling the standard form of the GL as given in [8]. The coefficients in the equation can be expressed in terms of the coefficients of the underlying system of partial differential equations (PDEs), therefore we study the dynamics of the GL for a wide range of parameters.

In this paper, we study solutions that become infinite in finite time, hence blow up. For these solutions, a contraction of the wave packet takes place, and simultaneously the amplitude grows and blows up. In nonlinear optics this phenomenon is called self-focusing, where it is related to an extreme increase of the field amplitude. In plasma physics it is called wave collapse.

In numerical simulations, sets of initial data for the GL were found such that the solutions indeed blow up, see $[5,10]$. In these simulations, radially symmetric, self-similar, multi-bump blowup solutions for the GL were found for $2<d<4$. Here, multi-bump is related to $|\Phi|$ having several maxima. In [5] an asymptotic analysis of these solutions was also given. Thereafter, the existence and local uniqueness of a radially symmetric, multi-bump, self-similar blowup solution was proved for $2<d<4$ in [11] by using geometric methods. These solutions only arise for dimensions $d>2$, since the dimension $d=2$ is the critical dimension for the GL; it distinguishes between integrable and blowup behaviour.

After setting $\varepsilon=0$ in the GL equation (1.1) it reduces to the well-known nonlinear Schrödinger equation (NLS). Blowup solutions of the NLS have already been studied extensively, see [16] for a survey, and for the most recent results, see [12] and references therein. The dimension $d=2$ is also critical for the NLS. We assume $\varepsilon \ll 1$ such that equation (1.1) is a small perturbation of the NLS.

In $[5,11]$, the radially symmetric, self-similar solutions were analysed using the method of dynamical rescaling, and we also use it here. This method exploits the asymptotically self-similar behaviour of the solutions. Following [5,11], space, time and $\Phi$ are scaled by the factors of a suitably chosen norm of the solutions, denoted by $L(t)$, which blows up at the singularity,

$$
\xi \equiv \frac{|x|}{L(t)}, \tau \equiv \int_{0}^{t} \frac{1}{L^{2}(s)} d s, u(\xi, \tau)=L(t) \Phi(x, t)
$$

The corresponding norm of the rescaled solution $u$ remains constant in time, and as a consequence, the rescaled problem is no longer singular. The rescaled solution $u$ satisfies

$$
i u_{\tau}+(1-i \varepsilon)\left[u_{\xi \xi}+\frac{d-1}{\xi} u_{\xi}\right]+(1+i b \varepsilon)|u|^{2} u+i a(\tau)(\xi u)_{\xi}=0
$$

where

$$
a=-L \frac{d L}{d t}=-\frac{1}{L} \frac{d L}{d \tau}
$$

It follows from the numerical simulations that self-similar blowup behaviour, with $L(t) \rightarrow$ 0 , arises when $a(\tau)$ is a positive constant and that $u$ can be written as $u(\xi, \tau)=e^{i w \tau} Q(\xi)$ for some positive constant $w$ that depends on the solution. After scaling $\tau$ with $\frac{1}{w}$, the 


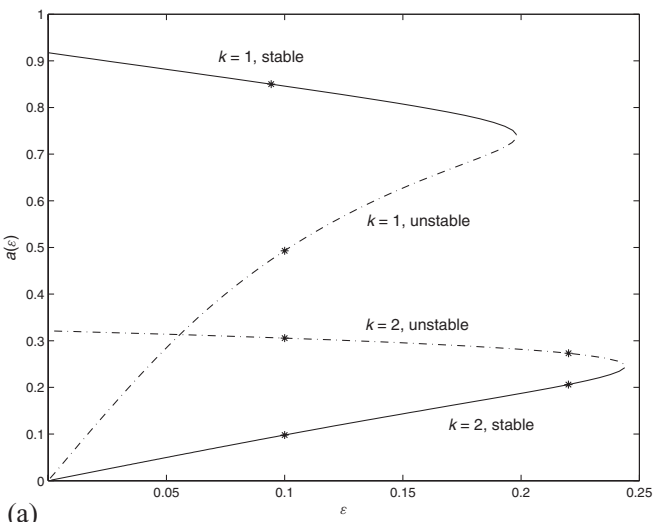

(a)

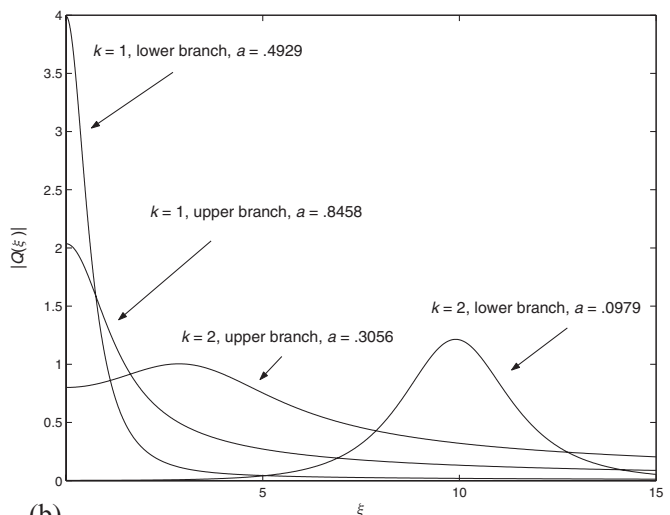

(b)

Figure 1. (a) The $k=1$ solution branch, the solutions with one maximum on $(-\infty, \infty)$, and the $k=2$ solution branch, the solutions with two maxima on $(-\infty, \infty)$, plotted in the $(\varepsilon, a)$-plane where $d=3$ and $b=0$. (b) Final-time profiles where the amplitude $|Q|$ is plotted as a function of the spatial variable $\xi$ for $\varepsilon=0.1$. The solutions correspond to the ${ }^{*} \mathrm{~s}$ in (a). This is a reproduction of Figures 1.1 and 1.2 in [5].

following equation for $Q$ can be obtained:

$$
(1-i \varepsilon)\left[Q_{\xi \xi}+\frac{d-1}{\xi} Q_{\xi}\right]-Q+i a(\xi Q)_{\xi}+(1+i b \varepsilon)|Q|^{2} Q=0 .
$$

Here the parameter $a$ plays the role of a nonlinear eigenvalue. In [10], the constant $w$ is left as an unknown; this does not affect the solutions since it can be scaled out.

Moreover, the initial and asymptotic conditions for $\Phi$, namely that $\Phi(x, 0)=\Phi_{0}(x)$ and that $|\Phi|$ vanishes as $|x| \rightarrow \infty$, lead to the following initial and asymptotic conditions for $Q$

$$
\begin{array}{ll}
Q_{\xi}(0)=0, & \operatorname{Im} Q(0)=0, \\
|Q(\xi)| \rightarrow 0 & \text { as } \quad \xi \rightarrow \infty .
\end{array}
$$

Here we have exploited the phase invariance of the equation to define the phase of $\Phi$ at the origin. Alternatively, we could have kept $w$ as an unknown in (1.3) and set $Q(0)=1$ as in [10].

First, we briefly summarise the results from the numerical simulations and asymptotic analysis as given in [5] for solutions where $|Q|$ has $k$ maxima on the real line. There $k$-solution branches are found in the $(\varepsilon, a)$-plane on which a symmetric solution with $k$ maxima on $(-\infty, \infty)$ exists for every $2<d<4$. In Figure 1(a), which is a reproduction of Figure 1.1 from [5], the branches for $k=1$ and $k=2$ where $b=0$ and $d=3$ are given. These branches correspond to symmetric solutions with one maximum at $\xi=0$ on the real line, $k=1$, and with two maxima, $k=2$, on the real line. The latter solutions $(k=2)$ have a minimum at $\xi=0$. The norm $|Q|$ of the solutions as found on the upper and lower parts of both branches at $\varepsilon=0.1$, the points indicated by the $*$ s, are given in Figure $1(\mathrm{~b})$.

Every $k$-solution branch consists of two parts, which coalesce. The solutions on the upper part of the branch are smooth perturbations of the solutions found for the NLS. Note that the intersection point of this part of the branch with $\varepsilon=0$ axis corresponds 
exactly to the NLS solutions. However, solutions on the lower part of the branch are not a simple perturbation of the solutions of the NLS. In this paper, we focus on solutions as found on the lower part of the $k$-solution branches.

Note that there is a clear distinction between solutions for which $k$ is even and for which it is odd. When $k$ is odd the $k$-solution has a maximum at $\xi=0$, on the other hand for even $k$ it has a minimum at $\xi=0$. In the numerical simulations, the maxima that lie away from $\xi=0$ are found for $a$ small in the range $\xi=\mathcal{O}\left(\frac{1}{a}\right)$ and just to the left of $\xi=\frac{2}{a}$, which is the point where the linearisation of (1.3) has a turning point. Thus, as $a \rightarrow 0^{+}$, all these maxima are created at $|\xi|=\infty$.

The existence and local uniqueness proof of [11] yields for every even $k$ two classes of $k$-bump solutions for $2<d<4$, and with $0<a \ll 1$, as long as certain relations between $a, d, b$ and $\varepsilon$ hold. Here we give the statement of the result and refer for more details to $[11]$.

Theorem 1.1 ([11]) For each $a>0$ sufficiently small, $2<d<4, b>0$ and conditions on $d, \varepsilon, b$ and $a$ as given in [11], there exists an $n_{0}(a)$ such that if $2 \leqslant n \leqslant n_{0}(a)$ and $n$ even, there exist $2 n$ locally unique $k=n$ solutions of equation (1.3) with initial conditions (1.4) and boundary conditions (1.5). These symmetric solutions consist of $n$ maxima on the real line where $\frac{n}{2}$ maxima are found on $0<\xi<\xi_{\max }$ with $\xi_{\max }=\frac{2-\sqrt{a}}{a}$. These maxima are strictly $\mathcal{O}\left(\log \left(\frac{1}{a}\right)\right)$ apart. Of the $2 n$ locally unique $k=n$ solutions, $n+1$ are characterised by the property that $\left|Q\left(\xi_{\max }\right)\right|$ is exponentially small; they are said to be of type L. For the other $n-1$, said to be of type $R,|Q|\left(\xi_{\max }\right)$ is strictly $\mathcal{O}\left(a^{\frac{3}{8}}\right)$.

Moreover, the sets of solutions of types $\mathrm{L}$ and $\mathrm{R}$ can be subdivided even further by distinguishing in the magnitude of $|Q|$ at $\xi=0$. There exist solutions for which $|Q(0)|$ is exponentially small in $a$ and solutions with $|Q(0)|$ not exponentially small but algebraically small in $a$. Both cases occur in both of the sets of types $\mathrm{L}$ and R. From the construction in [11], Section 6, we conclude that for the $k=n$ solutions of type L, two of the in total $n+1$ solutions satisfy the fact that $|Q(0)|$ is exponentially small, the rest of the $n-1$ solutions have a value of $|Q(0)|$, which is algebraically small. The $k=2$ solution of type $\mathrm{R}$ (there only exists one) has a value of $|Q(0)|$, which is exponentially small. For $n \geqslant 4$, the $n-1$ solutions of type $\mathrm{R}$ can be subdivided into two solutions with an exponentially small value of $|Q(0)|$, whereas for the rest of the $n-3$ solutions, $|Q(0)|$ is algebraically small.

The solutions constructed in [5] are of type L with $|Q(0)|$ exponentially small in $a$. In this paper, we perform an asymptotic analysis of the other type L solutions for which $|Q|$ at $\xi=0$ is not exponentially small in $a$ but algebraically small in $a$. This analysis differs on several major points from the one in [5] and the one for the NLS in [4]. Apart from another analysis, we also find that these solutions differ in more than just the magnitude of $|Q(0)|$. The $k=2$ solutions as constructed in [5] have a maximum at $\mathcal{O}\left(\frac{1}{a}\right)$, this is not true for solutions with $|Q(0)|$ algebraically small. An important conclusion from the analysis in this paper is that solutions that have a maximum close to $\mathcal{O}\left(\frac{1}{a}\right)$ also satisfy the fact that $|Q(0)|$ is exponentially small. In order for $|Q(0)|$ to be algebraically small, the maximum must lie well before $\mathcal{O}\left(\frac{1}{a}\right)$, hence $\xi_{\text {bump }} \ll \frac{1}{a}$ must hold for the position of

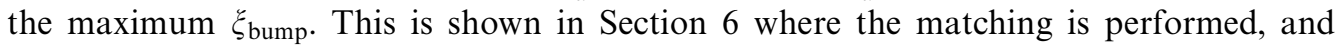


it indeed also follows from the geometric construction proof in [11]. More specifically, the analysis in [11] implies that the maximum of the solution lies to leading order at $\xi_{\text {bump }}=k_{1} \log \frac{1}{a}$ for some positive $k_{1}$. Here we find that this is indeed so and that $k_{1}=1$.

In this paper we construct solutions with $|Q(0)|$ algebraically small in $a$, which are of type $\mathrm{L}$ where the focus lies on the $k=2$ solution. Extension to solutions with more maxima in the interval can be done by extending the analysis in the so-called bump region as done in [4] for the NLS.

Recall that solutions found in the numerical simulations have a maximum at $\mathcal{O}\left(\frac{1}{a}\right)$. Hence, the solutions studied in this paper have so far not been found in numeric simulations. Also, the solutions of type $\mathrm{R}$ have, to our knowledge, not been found in numerical simulations or asymptotic analysis so far.

The stability of the type L solution with $A(0)$ exponentially small, which was found in [5], has been also analysed, see [14].

Remark 1.1 In [11], the analysis is performed for the case of $b>0$, although it can be extended to negative values of $b$. In this paper we do not assume that $b$ is positive but find in Section 5.1 that $b>-\frac{1}{4}$ must hold (as long as we assume that $\varepsilon>0$ ).

Remark 1.2 Choosing a non-integer dimension as done here is equivalent to taking $d=2$ and the power of the nonlinear term is equal to $2 \sigma$ for some positive $\sigma$.

\section{The main result and an overview of the approach}

In this section we state the main result of this paper and the method we use to obtain it. By applying asymptotic analysis, we construct the $k=2$ solution with $|Q|$ at $\xi=0$ algebraically small, and a maximum at $\frac{1}{\delta} \ll \frac{1}{a}$. This solution can only be constructed when the parameters in the problem satisfy the relations as specified in Main result For $a$ and $\varepsilon$ sufficiently small, $2<d<4$, there exists a $k=2$-solution branch on which a symmetric $k=2$ solution is found with a maximum at $\xi_{\text {bump }}=\frac{\kappa}{\delta} \ll \frac{1}{a}$ where

$$
\delta=\left(\log \frac{1}{a}\right)^{-1}
$$

and $\kappa=1$ to leading order. For this solution the value of $Q$ at $\xi=0$ is given by

$$
\begin{aligned}
Q(0) & =\delta^{\frac{1-d}{2}} \pi^{\frac{1}{2}}\left(\Gamma\left(\frac{d}{2}\right)\right)^{-1} 2^{\frac{4-d}{2}} \exp \left[-\frac{1}{\delta}\right], \\
& =\pi^{\frac{1}{2}}\left(\Gamma\left(\frac{d}{2}\right)\right)^{-1} 2^{\frac{4-d}{2}} a\left(\log \frac{1}{a}\right)^{\frac{d-1}{2}} .
\end{aligned}
$$

On this branch the parameters $a, b$ and $d$ satisfy

$$
4 a^{d-5}\left(\log \frac{1}{a}\right)^{2-d} e^{-\frac{\pi}{a}}=d-2-\frac{2 \varepsilon}{3 a}(1+4 b) .
$$


Hence, $Q(0)$ is indeed not exponentially small but algebraically small in $a$. The above choice of $\delta$ is indeed of the form as was expected from the existence proof of [11], see the Introduction.

In the region around the maximum at $\xi_{\text {bump }}=\frac{\kappa}{\delta}$ the leading order of the solution is given by

$$
Q(\xi)=e^{-\frac{i a \kappa}{2 \delta}\left(\xi-\frac{\kappa}{2 \delta}\right)} \sqrt{2} \operatorname{sech}\left(\xi-\frac{\kappa}{\delta}\right) .
$$

Note that this expression of the solutions - with $Q(0)$ asymptotically small - around the maximum differs not only in the position of the maximum with the solutions where $Q(0)$ is exponentially small for which the maximum lies at $\xi=\frac{\tilde{\kappa}}{a}$, [5] but the corresponding value of $Q$ at the maximum also differs. Here it is $\sqrt{2}$ whereas for the solutions constructed in [5] the value is expressed in terms of $\tilde{\kappa}$.

The relation (2.2) above between the parameters is quite special; it is a balance between an exponentially small term, the left-hand side, and an algebraically small term, the righthand side. Hence, the leading order of this expression is given by the right-hand side. In the asymptotic construction of the solutions in Section 5 this leading order reduction of (2.2) is indeed also found as a condition. The reduction gives an expression for the lower part of a $k=2$-branch similar to the $k=2$-branch given in Figure 1(a). However, the branch in Figure 1(a) is not the one given here; the solutions found in the numerics resulting in Figure 1(a) have a maximum at $\mathcal{O}\left(\frac{1}{a}\right)$, whereas the above branch (2.2) corresponds to solutions with $\xi_{\max }=\mathcal{O}\left(\frac{1}{\delta}\right) \ll \frac{1}{a}$. On the other hand, the leading order of the expression for the bifurcation curves is in both cases, whether the bump lies at $\mathcal{O}\left(\frac{1}{\delta}\right)$ or at $\mathcal{O}\left(\frac{1}{a}\right)$, the same. It is given by the right-hand side of (2.2). This might be one of the reasons that the solutions constructed in this paper have not yet been found in numerical simulations.

The above result is obtained by studying solutions of equation (1.3) with initial condition (1.4) and boundary condition (1.5). As in [5,11], we replace the boundary condition (1.5) by a local asymptotic condition at $\xi \rightarrow \infty$. For large $\xi$, it follows from the boundary condition (1.5), $|Q(\xi)| \rightarrow 0$, that the behaviour of the solutions is given by the dynamics of the linear part of equation (1.3)

$$
(1-i \varepsilon)\left[Q_{\xi \xi}+\frac{(d-1)}{\xi} Q_{\xi}\right]-Q+i a(\xi Q)_{\xi}=0 .
$$

For this equation, there exists a pair of linearly independent solutions for large $\xi$ given by

$$
Q_{1} \sim \xi^{-1-\frac{i}{a}}, \quad Q_{2} \sim \xi^{-\left(d-1-\frac{i}{a}\right)} e^{-i a \frac{\xi^{2}}{2}+\frac{a \xi^{2}}{2}} .
$$

Solution $Q_{2}$ is rapidly varying as $|\xi| \rightarrow \infty$, and has unbounded $H^{1}$-norm. The solutions we are looking for are slowly varying solutions, and hence their limiting profile for large $\xi$ is a multiple of $Q_{1}$. The asymptotic expressions for $Q_{1}$ and its derivative imply that

$$
\left|\xi Q_{\xi}+\left(1+\frac{i}{a}\right) Q\right| \rightarrow 0 \quad \text { as } \quad \xi \rightarrow \infty
$$

must hold, see [11]. In the NLS-limit this corresponds to solutions with finite Hamiltonian. From the fact that $Q_{1}$ decays at $\infty$, it follows that the boundary condition (1.5) is satisfied, 


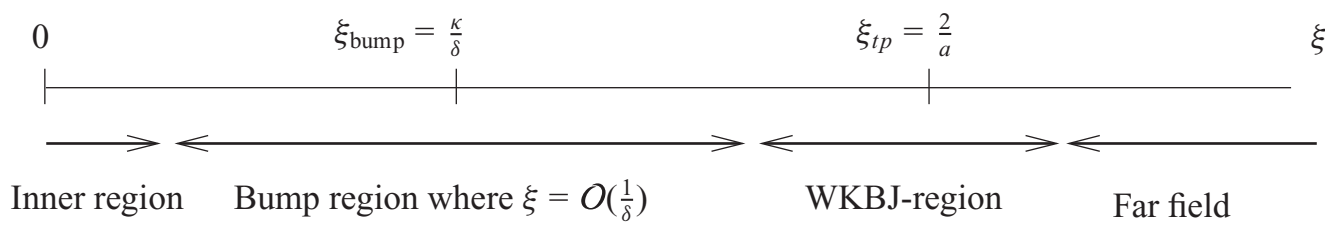

Figure 2. Different regions on the $\xi$-axis. As explained in Section 2, solutions are studied in these different regions by using asymptotic analysis, and thereafter the solutions are matched.

and therefore condition (1.5) can be omitted. Hence, from now on we study equation (1.3) combined with conditions (1.4) and (2.5).

The approach we take to study the solutions is to divide the positive real line, $\xi>0$, into several regions, see Figure 2, where we study the $Q$-equation (1.3) by using asymptotic analysis. Thereafter, we match the solutions as found in these different regions.

In one of the regions, the maximum is found at $\xi=\xi_{\text {bump }}=\frac{\kappa}{\delta}$, this is the so-called bump region. In this region, we find a sech-profile for the solution, see Section 5, leading indeed to a bump solution. The other regions lie to the left of the bump region where $\xi \ll \xi_{\text {bump }}$ - the so-called inner region - and to the right of the bump region where $\xi \gg \xi_{\text {bump }}$. Moreover, the region where $\xi \gg \xi_{\text {bump }}$ consists of the far field where $\xi \gg \frac{1}{a}$, and the region in between the bump region and far field where $\xi_{\text {bump }} \ll \xi \ll \frac{1}{a}$. In this latter part, a WKBJ-analysis needs to be used to match the bump region to the far field. See Figure 2 for a sketch of the $\xi$-axis where the different regions are indicated. The analysis in the inner region is performed in Section 4, and that of the far field can be found in Section 3. The matching of the inner solution to the left of the bump region is done in Section 6, and the bump solution is matched to the far field in Section 7 via a WKBJ-analysis.

Remark 2.1 The method we apply to obtain expression (2.1) for $\delta$ in terms of $a$ is quite unusual. This expression does not simply follow from the leading order analysis; it only arises after matching higher order terms of the solution in the inner region to the solution in the bump region, see Section 6 for a detailed explanation.

\subsection{Global estimates}

We can link the far field solution to the global behaviour of $Q$ via a rigorous result that relates the amplitude and phase of the solutions of (1.3). This relation is central in our final analysis, yielding the parameter branch (2.2), and we will state it here.

We decompose $Q$ into amplitude $A$ and gradient of the phase $\psi$ as

$$
Q(\xi)=A(\xi) \exp \left(i \int_{0}^{\xi} \psi(x) d x\right)
$$


the $Q$-equation (1.3) reduces to

$$
\begin{aligned}
A_{\xi \xi} & =\psi^{2} A-\frac{d-1}{\xi} A_{\xi}+A-A^{3}-\varepsilon\left(2 A_{\xi} \psi+A \psi_{\xi}+\frac{d-1}{\xi} A \psi\right)+a \xi A \psi, \\
\psi_{\xi} & =-2 \psi \frac{A_{\xi}}{A}-\frac{d-1}{\xi} \psi+\frac{\varepsilon}{A}\left(A_{\xi \xi}-A \psi^{2}+\frac{d-1}{\xi} A_{\xi}\right)-\frac{a}{A}\left(A+\xi A_{\xi}\right)-b \varepsilon A^{2} .
\end{aligned}
$$

As in [5], we obtain from this system the following integral equation for $\psi$.

Lemma 2.1 The phase $\psi$ and the amplitude A satisfy

$$
\psi+\frac{a \xi}{2}=\frac{1}{\xi A^{2}} \int_{0}^{\xi}(2-d) A^{2} \psi+\varepsilon x A\left(A_{x x}-A \psi^{2}+\frac{d-1}{x} A_{x}\right)-\varepsilon b x A^{4} d x .
$$

See Lemma 4.1 in [5] for a proof of this statement.

Once the structure of the solution is determined, this expression will be evaluated upon taking $\xi \rightarrow \infty$, in Section 8 . This yields the resulting relation between the parameters as given in (2.2).

\section{The far field behaviour when $\xi \gg \frac{1}{a}$}

In this section, we consider the behaviour of $Q$ for $\xi \gg \frac{1}{a}$. The boundary condition (1.5) requires that $|Q|$ is very small in this range of $\xi$-values, and therefore equation (1.3) can be approximated by the linearised equation

$$
(1-i \varepsilon)\left(Q_{\xi \xi}+\frac{d-1}{\xi} Q_{\xi}\right)-Q+i a(\xi Q)_{\xi}=0 .
$$

Using the Liouville transformation $Q(\xi)=e^{-\frac{i a}{4(1-i \varepsilon)} \xi^{2}} \xi^{\frac{1-d}{2}} W$, this equation can be written in a self-adjoint form, leading to the parabolic cylinder equation for $W$

$$
W_{\xi \xi}+W\left(\frac{a^{2} \xi^{2}}{4}-1\right)=0
$$

for $0<a, \varepsilon \ll 1$ and $\xi \gg 1$. The solutions to this equation change type at the turning point $\xi=\frac{2}{a}$, admitting exponentially decaying solutions for $\xi<\frac{2}{a}$ and polynomially decaying solutions for $\xi>\frac{2}{a}$.

In the far field, where $\xi \gg \frac{2}{a}$, there exist (complex) constants $\mu, v$ such that as for $a$ and $\varepsilon$ small, solutions are given by

$$
Q(\xi) \sim \mu \xi^{-1-i / a}\left(1+\mathcal{O}\left(\frac{1}{a \xi^{2}}\right)\right)
$$

or

$$
Q(\xi) \sim v \xi^{1-d+i / a} e^{-i a \xi^{2} / 2} e^{a \varepsilon \xi^{2} / 2}\left(1+\mathcal{O}\left(\frac{1}{a \xi^{2}}\right)\right),
$$

see [5]. The first of these two solutions is slowly varying and decaying, whereas the second is rapidly varying and growing when $\varepsilon>0$. Therefore, only this first solution satisfies the 
local condition for $Q(\xi)$ given in (2.5), implying that in the far field the solution is given by (3.3).

In the matching procedure in Section 7, we return to the $W$-equation (3.2) and analyse the solutions close to the turning point $\xi=\frac{2}{a}$ by using the WKBJ-method.

\section{Solutions in the inner region}

In this section, we study the solutions in the inner region where $|Q| \ll 1$ and $\xi \ll 1$. Again, the linearised equation (2.3) gives the leading order dynamics. Introducing the rescaling $Q(\xi)=e^{-\frac{i a}{4 \sqrt{1-i \varepsilon}} \xi^{2}} W(y)$ with $y=\frac{\xi}{\sqrt{1-i \varepsilon}}$, equation (2.3) is rescaled to

$$
W_{y y}+\frac{d-1}{y} W_{y}+\left(-1+\frac{i a}{2}\right) W=0,
$$

to leading order. After introducing $z=2 y$ and $W=R e^{-y}$ we obtain

$$
z R_{z z}+(d-1-z) R_{z}+\left(\frac{1-d}{2}+\frac{i a}{8} z\right) R=0
$$

which reduces to leading order - without the second $R$-term - to the canonical Kummer equation. For this Kummer equation, there exist two independent solutions denoted by $M\left(a_{1}, b_{1}, z\right)$ (alternatively, denoted as the confluent hypergeometric function ${ }_{1} F_{1}\left(a_{1} ; b_{1} ; z\right)$ ) and $U\left(a_{1}, b_{1}, z\right)$, where $a_{1}=\frac{d-1}{2}$ and $b_{1}=d-1$. Hence, to leading order, a solution to (4.2) is given by the linear combination

$$
R(z)=\alpha_{i n} M\left(\frac{d-1}{2}, d-1, z\right)+c_{U} U\left(\frac{d-1}{2}, d-1, z\right),
$$

where $\alpha_{i n}$ and $c_{U}$ are constants. However, the function $U$ is singular at $z=0$ and thus we must set $c_{U}=0$.

Rescaling back to the original variables, the solution to the linearised $Q$ equation (2.3) is given, to leading order, by

$$
Q_{i n}(\xi)=\alpha_{i n} e^{-\frac{i a}{4 \sqrt{1-i \varepsilon}} \xi^{2}} e^{-\frac{\xi}{\sqrt{1-i \varepsilon}}} M\left(\frac{d-1}{2}, d-1, \frac{2 \xi}{\sqrt{1-i \varepsilon}}\right),
$$

for some constant $\alpha_{i n}$. Then, it can be verified by direct calculation and using the fact that $\frac{d}{d z}(M(a, b, z))=\frac{a}{b} M(a+1, b+1, z)$ that the boundary condition $Q_{\xi}(0)=0$ is indeed satisfied.

Using that $M\left(a_{1}, b_{1}, 0\right)=1$ gives that $Q(0)=\alpha_{i n}$. Thus, the condition (1.4) that $Q(0)$ is real implies that $\alpha_{i n}$ must be real. Also, recall that we are constructing solutions for which $|Q|$ at $\xi=0$ is algebraically small in $a$. This implies that $Q(0)=\alpha_{i n}$ has to be algebraically small in $a$ (and not exponentially small).

Remark 4.1 In [5] it is shown that in the inner region where $\xi \ll 1$, the amplitude $|Q|$ of the solution satisfies to leading order the so-called ground-state equation which admits a discrete set of exponentially decaying solutions. One of them being $|Q|=0$, and another 
the ground-state solution (or the Townes soliton). Here we concentrate on solutions close to $|Q|=0$ because we require $Q(0)$ to be small.

\section{Asymptotic analysis of the solutions in the bump region}

Now we study the solution in the region where the maxima lie: the bump region. As was mentioned in the Introduction, we will concentrate our analysis on solutions with one maximum in this region.

In this section we assume a balance between the terms in the $Q$ equation (1.3) that contain the parameter $\varepsilon$, representing the perturbation away from the NLS, and the small parameter $a$, and therefore set

$$
\varepsilon=K a,
$$

where $K>0$ and $K=\mathcal{O}(1)$. Note that this choice corresponds to an analog of the lower branch of the $k=2$ solutions in Figure 1(a), although the solutions given in that figure are not the solutions constructed in this paper, see the Introduction.

As has been already explained in detail in the Introduction, we assume that the maximum of the solution is found in the region where $\xi=\mathcal{O}\left(\frac{1}{\delta}\right)$ and $a \ll \delta \ll 1$. More specifically, we assume that the peak is located at the point

$$
\xi_{\text {bump }}=\frac{\kappa}{\delta}
$$

with $a \ll \delta \ll 1$, and seek to determine $\kappa$. In other words, $\kappa$ is defined such that the maximum of $|Q|$ lies exactly at $\frac{\kappa}{\delta}$. Furthermore, since $\delta$ is still free to be chosen, we can fix the leading order of $\kappa$ to be equal to 1 , hence we take $\kappa=1+$ hot. Note that this is possible since $\delta$ is not a parameter in the equation (contrary to $\varepsilon$ ).

Now we focus on the region around the maximum and rescale the $Q$-equation (1.3) by setting

$$
\xi=\frac{\kappa}{\delta}+s
$$

This leads to

$$
(1-i a K)\left[Q_{s s}+\delta \frac{d-1}{\kappa+\delta s} Q_{s}\right]-Q+i \frac{a}{\delta}((\kappa+\delta s) Q)_{s}+(1+i a b K)|Q|^{2} Q=0 .
$$

Also, we expand both of the parameters $\kappa$ and $K$ in terms of $\delta$ and $a$ :

$$
\begin{aligned}
\kappa & =1+\delta \kappa_{\delta}+\cdots+\frac{a}{\delta} \kappa_{\frac{a}{\delta}}+\cdots+a \kappa_{a}+\cdots \\
K & =K_{0}+\delta K_{\delta}+\cdots+\frac{a}{\delta} K_{\frac{a}{\delta}}+\cdots+a K_{a}+\cdots
\end{aligned}
$$

where we fix the leading order term of $\kappa$ identical to 1 . Moreover, we require that the solution $|Q|$ takes on a localised form that is independent of $\delta$ and $a$, provided that they both are small. Up to this point in the analysis we have not assumed any condition on the relation between powers of $\delta$ and $a$ (apart from $a \ll \delta$ ). To stress this, we have written the expansions in the above form. 
We start the analysis of the $Q$-equation (5.3) by reducing the self-adjoint $Q_{s}$ terms in this equation as much as possible. By introducing rescaling in the phase of $Q$

$$
Q(s)=e^{-\frac{i a \kappa}{2 \delta} s} S(s)
$$

the leading order self-adjoint terms are reduced and become of higher order. This leads to

$$
\begin{aligned}
& S_{s s}-S+|S|^{2} S=S\left[-i a\left(1-\frac{\kappa(d-1)}{2(\kappa+\delta s)}(1-i a K)\right)-\frac{a^{2} \kappa}{4 \delta^{2}}(\kappa(1+i a K)+2 \delta s)\right] \\
& +S_{S}\left[\frac{\delta(1-d)}{\kappa+\delta s}(1-i a K)-i a s+\frac{a^{2} \kappa}{\delta} K\right]+i a K S_{s s}-i a b K|S|^{2} S .
\end{aligned}
$$

We now express $S(s)$ as an asymptotic series in $a$ and $\delta$

$$
S(s)=\tilde{K}_{0}\left[S_{0}(s)+\delta S_{\delta}(s)+\cdots+\frac{a^{2}}{\delta^{2}} S_{\frac{a^{2}}{\delta^{2}}}(s)+\cdots+a S_{a}(s)+\cdots\right],
$$

where $\tilde{K}_{0}$ is a complex constant with $\left|\tilde{K}_{0}\right|=1$, and $S_{0}$ is a real function; this can be done because of the phase invariance of the equation.

Note that the above expansion for $S$ has to be a consistent asymptotic expression in the bump region. Therefore, we need, for example, that $\delta \operatorname{Re}\left(S_{\delta}\right) \ll S_{0}$ and $\operatorname{Im}\left(S_{\delta}\right) \rightarrow 0$ for $s \rightarrow \pm \infty$ (since $S_{0}$ is real) in the bump region. Here $\operatorname{Re}(S), \operatorname{resp} \operatorname{Im}(S)$, is the real, resp imaginary, part of $S$. Similar relations need to hold for the higher order terms. The most common and easy way to satisfy both conditions is to assume that $\operatorname{Re}\left(S_{\delta}\right) \rightarrow 0$ for $s \rightarrow \pm \infty$ holds as well. It will turn out that this is not possible for both $s \rightarrow+\infty$ and $s \rightarrow-\infty$, and therefore we will restrict the bump region to that part where $\delta \operatorname{Re}\left(S_{\delta}\right) \ll S_{0}$. Note that this is quite unusual when applying the method of asymptotic analysis. However, it is not quite unexpected because the solution in the inner region - to which this bump solution needs to be matched - contains for $\xi \gg 1$ an exponentially decaying term and an exponentially growing term, see Section 6.

The assumption that the maximum of $|Q|$ lies at $\xi=\frac{\kappa}{\delta}$, hence at $s=0$ gives that

$$
\begin{aligned}
0 & =\left(|Q|^{2}\right)_{\xi}\left(\xi=\frac{\kappa}{\delta}\right)=\left(|Q|^{2}\right)_{s}(s=0)=2|Q||Q|_{s}(s=0) \\
& =\left.2\left(\operatorname{Re}(S) \frac{d}{d s}(\operatorname{Re} S)+\operatorname{Im}(S) \frac{d}{d s}(\operatorname{Im} S)\right)\right|_{s=0} .
\end{aligned}
$$

This leads to conditions on the derivatives of the terms in the expansion for $S$.

Substituting the expansion of $S$ (5.7) into the $S$-equation (5.6) leads at the $\mathcal{O}(1)$-level to the following equation for $S_{0}$

$$
S_{0, s s}-S_{0}+\left|S_{0}\right|^{2} S_{0}=0
$$

As mentioned before, we assume the solution $S_{0}$ of (5.9) to be real, so we find

$$
S_{0}(s)=\sqrt{2} \operatorname{sech}(s) .
$$


Rewriting this in terms of the original variable $\xi$ gives the following leading order expression of the $Q$-solution in the bump region

$$
Q(\xi)=e^{-\frac{i a \kappa}{2 \delta}\left(\xi-\frac{\kappa}{\delta}\right)} \tilde{K}_{0} \sqrt{2} \operatorname{sech}\left(\xi-\frac{\kappa}{\delta}\right),
$$

where $\tilde{K}_{0} \in \mathbf{C}$ and $\left|\tilde{K}_{0}\right|=1$.

\subsection{Higher order terms in the bump region}

Now we study the equations for the higher order terms in the expansion of $S$; this analysis yields an expression for the leading order terms of $K$ and gives the range of $s$-values where expansion (5.7) is asymptotic, thereby restricting the bump region.

The $\mathcal{O}(\delta)$-terms in equation (5.6) lead to the following equation for $S_{\delta}$ :

$$
S_{\delta, s s}-S_{\delta}+S_{0}^{2} \bar{S}_{\delta}+2\left|S_{0}\right|^{2} S_{\delta}=(1-d) S_{0, s}
$$

Splitting $S_{\delta}$ into complex and real parts as $S_{\delta}=t_{\delta}+i v_{\delta}$, we find

$$
\begin{aligned}
t_{\delta, s s}-t_{\delta}+3 S_{0}^{2} t_{\delta} & =(1-d) S_{0, s}=f_{\delta} \\
v_{\delta, s s}-v_{\delta}+S_{0}^{2} v_{\delta} & =0
\end{aligned}
$$

where $f_{\delta}$ is defined as the right-hand side of the equation for $t_{\delta}$.

The equation for $t_{\delta}$ has two linearly independent solutions, which are given by $\psi_{1}(s):=$ $S_{0, s}$ and $\psi_{2}(s):=S_{0, s} \int^{s} \frac{1}{S_{0, x}^{2}} d x$. Here the solutions $\psi_{1}$ and $\psi_{2}$ are constructed in such a way that the Wronskian is equal to 1 . Furthermore, $\psi_{1}$ is odd and localised, and $\psi_{2}$ is even and unbounded. Using the method of variation of constants, the general solution of the $t_{\delta}$-equation is then given by

$$
t_{\delta}=A_{\delta} \psi_{1}+B_{\delta} \psi_{2}-\psi_{1} \int_{0}^{s} \psi_{2} f_{\delta} d x+\psi_{2} \int_{0}^{s} \psi_{1} f_{\delta} d x
$$

for some constants $A_{\delta}, B_{\delta}$.

From the condition (5.8) that $|Q|$ obtains its maximum at $\xi=\frac{\kappa}{\delta}$, we find that $t_{\delta, s}(0)=0$, which in turn implies that $A_{\delta}=0$. After evaluating the integrals, $t_{\delta}$ is given by

$$
t_{\delta}=-\frac{\operatorname{sech}^{2}(s)}{24 \sqrt{2}}\left[3 B_{\delta}(\cosh (3 s)-9 \cosh (s))+4\left((d-1)(1-\cosh (2 s))+9 B_{\delta}\right) \sinh (s)\right] .
$$

This expression grows exponentially as $|s| \rightarrow \infty$, more specifically,

$$
t_{\delta} \rightarrow-\frac{e^{|s|}}{6 \sqrt{2}}\left[\frac{3}{2} B_{\delta} \pm(1-d)\right]
$$

as $s \rightarrow \pm \infty$. Unfortunately, the constant $B_{\delta}$ cannot be chosen such that $t_{\delta}$ decays to zero for both $s \rightarrow+\infty$ and $s \rightarrow-\infty$. However, as explained above, for a correct asymptotic analysis we do need that the expansion for $S$ (5.7) is an asymptotic expansion. Hence, the condition $\delta t_{\delta} \ll S_{0}$ must be satisfied for all $s$ in the bump region. Now we can still 
choose $B_{\delta}$ and with this choice make certain that $t_{\delta}$ decays to zero either as $s \rightarrow+\infty$ or as $s \rightarrow-\infty$. It turns out (from the matching in Section 6 ) that it is more convenient to choose

$$
B_{\delta}=\frac{2(d-1)}{3}
$$

so that $t_{\delta}$ decays to zero for $s \rightarrow+\infty$. This comes from the fact that the inner solution as found in Section 4 contains a higher order term which is exponentially growing, see expression (6.4), whereas the higher order terms in the solution found in the WKBJ-region are exponentially small, see (7.5). Thus, we can match the exponentially growing term to the left and not to the right. For negative values of $s$ we restrict to that part of the bump region where $\delta t_{\delta} \ll S_{0}$ is satisfied. This implies that the bump region can only contain those negative $s$-values for which $|s| \ll \log \left(\frac{1}{\delta}\right)$.

Now we analyse the equation for $v_{\delta}$. Two linearly independent solutions to the $v_{\delta}$ equation are given by $\phi_{1}(s):=S_{0}$ and $\phi_{2}(s):=S_{0} \int_{0}^{s} \frac{1}{S_{0}^{2}} d x$, where $\phi_{1}$ and $\phi_{2}$ are again constructed in such a way that the Wronskian is identical to 1 . Furthermore, $\phi_{1}$ is even and localised, and $\phi_{2}$ is odd and unbounded. Then the general solution to the $v_{\delta}$-equation is given by the linear combination

$$
v_{\delta}=C_{\delta} \phi_{1}+D_{\delta} \phi_{2}
$$

for some constants $C_{\delta}, D_{\delta}$. Now, since $v_{\delta}$ must decay to zero as $s \rightarrow \pm \infty$ and $\phi_{2}$ is unbounded, we need to set $D_{\delta}=0$.

The analysis at the $\mathcal{O}\left(\delta^{l}\right)$-level for $l \geqslant 2$ can be performed in a similar way, where in the restricted bump region (where for $s<0,|s| \ll \log \frac{1}{\delta}$ ) the expansion for $S$ is indeed asymptotic.

It turns out that in order to obtain an expression for the leading order term of $K, K_{0}$, in terms of the parameters $d$ and $b$, we need to study the equation at $\mathcal{O}(a)$ level. After substituting expression (5.6) into equation (5.7), collecting the $\mathcal{O}(a)$-terms and separating into real and imaginary parts, with $S_{a}=t_{a}+i v_{a}$, the equations are given by

$$
\begin{aligned}
& t_{a, s s}-t_{a}+3 S_{0}^{2} t_{a}=0, \\
& v_{a, s s}-v_{a}+S_{0}^{2} v_{a}=\frac{d-3}{2} S_{0}-s S_{0, s}+K_{0} S_{0, s s}-b K_{0} S_{0}^{3}=g_{a},
\end{aligned}
$$

where the right-hand side of the $v_{a}$-equation is denoted by $g_{a}$. Similar to the above analysis for $t_{\delta}$, we can solve both equations by using the solutions of the homogeneous equation and the method of variations of constants. Thereafter, the requirement that the expansion for $S$ (5.7) must be asymptotic, yields an expression for $K_{0}$.

From the analysis of the $t_{a}$-equation, yielding $t_{a}=A_{a} \psi_{1}+B_{a} \psi_{2}$, we do not find any condition on the parameters in the problem, therefore we omit it here, and continue with the study of the solution of the $v_{a}$-equation.

The method of variation of constants yields

$$
v_{a}=C_{a} \phi_{1}+D_{a} \phi_{2}-\phi_{1} \int_{0}^{s} \phi_{2} g_{a} d x+\phi_{2} \int_{0}^{s} \phi_{1} g_{a} d x
$$


Now we need for an asymptotic expansion that $\left|v_{a}\right| \rightarrow 0$ as $|s| \rightarrow \infty$. Since $\phi_{2}$ is exponentially growing, this will result in conditions on the parameters. By using that both $\phi_{1}$ and $g_{a}$ are even, we find from assuming that $\left|v_{a}\right| \rightarrow 0$ as $|s| \rightarrow \infty$ that $D_{a}=0$. Moreover, the condition

$$
0=\int_{0}^{\infty} \phi_{1} g_{a} d x=\int_{0}^{\infty} \phi_{1}\left[\frac{d-3}{2} S_{0}-s S_{0, s}+K_{0} S_{0, s s}-b K_{0} S_{0}^{3}\right] d x,
$$

must be satisfied. Determining the integrals, this yields

$$
d-2-\frac{2}{3} K_{0}(1+4 b)=0
$$

Hence, we find that $K_{0}$ can be expressed in terms of $b$ and $d$, as

$$
K_{0}=\frac{3(d-2)}{2(1+4 b)}
$$

In Section 8, we find to leading order the same expression for $K_{0}$ as here by using the integral condition (2.7) that was introduced in Section 2.1.

Recall that the coefficient $K_{0}$ determines the (leading order) relation between $\varepsilon$ and $a$ by $\varepsilon=K_{0} a+$ hot. From the fact that both $\varepsilon$ and $a$ are positive, we conclude that $K_{0}$ must be positive, and hence $b>-\frac{1}{4}$ ( since $d>2$ ). Moreover, for $b$ close (in $a$ ) to $b=-\frac{1}{4}$, the above analysis can only be performed with the restriction that $d-2$ is small.

\section{Matching the bump region to the inner region}

In the subsequent sections, we match the solutions as found in Sections 3-5. In this section, we match the solution in the inner region to the left-hand side of the solution in the bump region, see Figure 2. Thereafter, in Section 7, we match the right-hand side of the bump region to the far field by using a WKBJ-method to construct an asymptotic solution on the real line.

By introducing $y=-\xi+\frac{\kappa}{\delta}$, where $y>0,1 \ll y \ll \log \frac{1}{\delta}$, we write the inner solution and the bump solution in terms of this new variable $y$, which represents the region where they are both valid. Then the solution in the bump region is, to leading order, given by

$$
Q(y)=\tilde{K}_{0} \sqrt{2} e^{i \frac{a \kappa}{2 \delta} y} e^{-y},
$$

where $\left|\tilde{K}_{0}\right|=1$. By using the asymptotics for the Kummer solution, the solution in the inner region becomes (in terms of $y$ )

$$
Q_{i n}(y)=\alpha_{i n} \pi^{-\frac{1}{2}} \Gamma\left(\frac{d}{2}\right) 2^{\frac{d-3}{2}}\left(\frac{\kappa}{\delta}-y\right)^{\frac{1-d}{2}} e^{-i \frac{a \kappa^{2}}{4 \delta^{2}}+i \frac{a \kappa y}{2 \delta}}\left[e^{-y+\frac{\kappa}{\delta}}+e^{\frac{i \pi(d-1)}{2}} e^{y-\frac{\kappa}{\delta}}\right] .
$$


The solution (6.1) and the leading order term - the first term - in (6.2) match perfectly upon choosing

$$
\begin{aligned}
& \tilde{K}_{0}=\exp \left[-i \frac{a \kappa^{2}}{4 \delta^{2}}\right] \\
& \alpha_{i n}=\delta^{\frac{1-d}{2}} \pi^{\frac{1}{2}}\left(\Gamma\left(\frac{d}{2}\right)\right)^{-1} 2^{\frac{4-d}{2}} \exp \left[-\frac{1}{\delta}\right],
\end{aligned}
$$

where we use that $Q(0)$, and hence $\alpha_{i n}$, must be real, see Section 4. Also, we use the fact that $\kappa=1+$ hot - recall that $\xi_{\text {bump }}=\frac{\kappa}{\delta}$.

The aim of the analysis in this paper is to construct solutions for which $Q(0)$, and hence $\alpha_{i n}=Q(0)$, is algebraically small in $a$ (and not exponentially small). Then the above expression for $\alpha_{i n},(6.3)$, is only algebraically small when $\exp \left[-\frac{1}{\delta}\right]$ is algebraically small in $a$. From this we can conclude that $\delta$ must be chosen such that $\delta \gg a^{l}$ for every $l>0$.

Now it can also be explained that taking the bump of the solution at $\xi_{\text {bump }}=\mathcal{O}\left(\frac{1}{a}\right)$, as in [5], immediately implies that $Q(0)$ is exponentially small. Namely, choosing $\xi_{\text {bump }}=\mathcal{O}\left(\frac{1}{a}\right)$ corresponds to setting $\delta=a$ in (6.3). This gives that $\alpha_{i n}$, and hence $Q(0)$, are exponentially small.

In the subsequent analysis we will determine an expression for $\delta$ in terms of $a$. In order to obtain such an expression (6.5) for $\delta$, we focus on the second term in expansion (6.2) of the solution in the inner region.

With the above choice of $\alpha_{i n}$, expansion (6.2) becomes

$$
Q_{i n}(y)=\sqrt{2} \tilde{K}_{0} e^{i \frac{a k y}{2 \delta}}\left[e^{-y}+e^{-\frac{2}{\delta}} e^{\frac{i \pi(d-1)}{2}} e^{y}\right] .
$$

In the matching procedure, the second term in this expression must also be matched to a (higher order) term of the solution valid in the bump region. Since the term is of $\mathcal{O}\left(e^{-\frac{2}{\delta}}\right)$, this can only be done for suitable choices of $\delta$.

The expansion in the bump region contains terms of $\mathcal{O}\left(\delta^{l_{1}}\right), \mathcal{O}\left(\frac{a^{m_{1}}}{\delta^{m_{2}}}\right)$ and $\mathcal{O}\left(a^{l_{2}}\right)$, where $l_{i}$ and $m_{i}$ are positive integers, $m_{2} \leqslant m_{1}$ and $m_{1} \geqslant 2$. Now we must match the $\mathcal{O}\left(e^{-\frac{2}{\delta}}\right)$-term to one of these terms. Note that $\mathcal{O}\left(e^{-\frac{2}{\delta}}\right)$-term cannot be matched to a $\mathcal{O}\left(\delta^{l_{1}}\right)$-term. In case we match the $\mathcal{O}\left(e^{-\frac{2}{\delta}}\right)$-term to either a $\mathcal{O}\left(\frac{a^{m_{1}}}{\delta^{m_{2}}}\right)$-term or a $\mathcal{O}\left(a^{l_{2}}\right)$-term (where $l_{2} \geqslant 1, m_{2} \leqslant m_{1}$, $m_{1} \geqslant 2$ ), this results, in both cases, to the following leading order expression,

$$
\delta=\frac{1}{c_{\delta} \log \frac{1}{a}},
$$

where $c_{\delta}$ is a positive constant. Here the higher order terms that arise by equating $e^{-\frac{2}{\delta}}$ with a $\mathcal{O}\left(\frac{a^{m_{1}}}{\delta^{m_{2}}}\right)$-term in the resulting expression for $\delta$ are incorporated in the higher order terms in the expression for $\kappa(5.4)$.

Upon choosing $\delta$ as given in expression (6.5), we find that $\mathcal{O}\left(e^{-\frac{2}{\delta}}\right)=a^{2 c_{\delta}}$. We will match this term to a $\mathcal{O}\left(a^{l_{2}}\right)$-term in the bump region, and therefore we need to choose $2 c_{\delta}$ equal to a positive integer. Thus, $2 c_{\delta}=N$, where $N \in \mathbf{N}$.

Now we show that matching to the $\mathcal{O}(a)$-term $S_{a}$ in the bump region leads to a contradiction. The reason for this is that the second term in (6.4) is exponentially growing in $y$, but $S_{a}=t_{a}+i v_{a}$ is not growing. First, we study the behaviour of the function $v_{a}$. 
In Section 5.1 we assume that $v_{a}$ decays to zero as $s \rightarrow \pm \infty$. There this leads to the condition (5.13) for $K_{0}$. If we discard the assumption that $v_{a}$ decays as $s \rightarrow \pm \infty$, and hence, this condition (5.13), the matching can indeed be performed. However, in Section 8 , the integral condition (8.4) is used to determine a relation between the parameters, and there we rediscover the same expression (5.13) for $K_{0}$ as in Section 5.1. This means that $v_{a}$ indeed decays exponentially to zero as $s \rightarrow \pm \infty$ and cannot be matched to the second term in (6.4).

Now we analyse whether we can match to the real part, $t_{a}$, of $S_{a}$. For this we need that the second term in (6.4) is also real (to leading order). From this condition we obtain that $\frac{\pi(d-2)}{2}=k \pi, k \in \mathbf{Z}$, to leading order. Hence, since $2<d<4$, this implies that $d$ must be either close to $d=2$ or close to $d=4$. We do not want to impose this extra restriction, therefore matching to the $S_{a}$-term is not possible.

Concluding, we should match the second term in (6.4) to the $\mathcal{O}\left(a^{2}\right)$-term in the expression for $S$ (5.7): $S_{a^{2}}=t_{a^{2}}+i v_{a^{2}}$. Therefore, we must choose

$$
c_{\delta}=1
$$

so that the matching can indeed be performed.

The above analysis results in the expression

$$
\delta=\left(\log \frac{1}{a}\right)^{-1} .
$$

Also, this gives

$$
Q(0)=\alpha_{i n}=\pi^{\frac{1}{2}}\left(\Gamma\left(\frac{d}{2}\right)\right)^{-1} 2^{\frac{4-d}{2}} a\left(\log \frac{1}{a}\right)^{\frac{d-1}{2}},
$$

which indeed is algebraically small in $a$.

\section{Matching the bump solution to the far field solution}

In this section, we match the bump region to the far field. Recall that the far field solution determined in Section 3 is valid as long as $\xi \gg \frac{2}{a}$. In order to match the solution in the far field to the solution in the bump region, we need to track the solution through the region that lies between the bump region, where $\xi=\mathcal{O}\left(\frac{1}{\delta}\right)$, and the region where the far field solution is valid (where $\xi \gg \frac{2}{a}$ ). In this region, the dynamics of the solution is described by the parabolic cylinder equation (3.2) as found in Section 3. This equation has a turning point at $\xi=\xi_{t p}=\frac{2}{a}$ and exhibits exponential behaviour to the left, and parabolic behaviour to the right of this turning point. We apply the WKBJ-method to equation (3.2) to obtain the solution in this region. Close to $\xi_{t p}$, equation (3.2) reduces to the Airy equation, see Section 7.1.

Upon introducing $x=\frac{a \xi}{2}$, equation (3.2) becomes

$$
W_{x x}-\frac{4}{a^{2}}\left[1-x^{2}\right] W=0 .
$$


Note that in the rescaled variable the turning point corresponds to $x_{t p}=1$. Using the WKBJ-method we find that the solution is given by

$$
W=\left(1-x^{2}\right)^{-\frac{1}{4}}\left[C_{-} e^{-\frac{2}{a} \int_{1}^{x} \sqrt{q(s)} d s}+C_{+} e^{\frac{2}{a} \int_{1}^{x} \sqrt{q(s)} d s}\right]
$$

where $q(x)=1-x^{2}$. The integral in the exponential can be determined as

$$
F(x)=\int_{1}^{x} \sqrt{q(s)} d s=\frac{1}{2} x \sqrt{1-x^{2}}+\frac{1}{2} \arcsin x-\frac{\pi}{4},
$$

and thus, the WKBJ-solution is, in terms of the original variables, given by

$$
Q(\xi)=e^{-\frac{i a}{4(1-i \varepsilon)} \xi^{2}} \xi^{\frac{1-d}{2}}\left(1-\frac{a^{2} \xi^{2}}{4}\right)^{-\frac{1}{4}}\left[C_{-} e^{-\frac{2}{a} F\left(\frac{a \xi}{2}\right)}+C_{+} e^{\frac{2}{a} F\left(\frac{a \xi}{2}\right)}\right] .
$$

Now we match this solution to both bump solution and far field solution. First, we match the bump solution on the right-hand side to the WKBJ-solution. For that we study the solutions in the region where they are both valid; we introduce $\xi=\frac{\kappa}{\delta}+y$, with $y>0,1 \ll y \ll \frac{1}{\delta}$. Then the solution in the bump region is given by

$$
Q(y)=\tilde{K}_{0} \sqrt{2} e^{-\frac{i a \kappa}{2 \delta} y} e^{-y}
$$

and the WKBJ-solution reduces to

$$
Q(y)=e^{-\frac{i a \kappa^{2}}{4 \delta^{2}}} e^{-\frac{i a \kappa}{2 \delta} y}\left(\frac{1}{\delta}\right)^{\frac{1-d}{2}}\left[C_{-} e^{-y-\frac{\kappa}{\delta}+\frac{\pi}{2 a}}+C_{+} e^{\frac{\kappa}{\delta}+y-\frac{\pi}{2 a}}\right],
$$

to leading order. Since $\frac{\pi}{2 a} \gg \frac{\kappa}{\delta}$, the second term in this expression is exponentially small, and the solutions can be matched upon choosing

$$
\tilde{K}_{0} \sqrt{2}=e^{-\frac{i a \kappa^{2}}{4 \delta^{2}}}\left(\frac{1}{\delta}\right)^{\frac{1-d}{2}} C_{-} e^{-\frac{\kappa}{\delta}+\frac{\pi}{2 a}}
$$

Combining this with the expression as obtained for $\tilde{K}_{0}$ in equation (6.3), and the fact that $\kappa=1+$ hot, yields

$$
C_{-}=\sqrt{2} \delta^{\frac{1-d}{2}} e^{\frac{1}{\delta}-\frac{\pi}{2 a}}
$$

\subsection{The Airy equation}

At $\xi=\xi_{t p}=\frac{2}{a}$, equation (3.2) has a turning point, hence close to this point the WKBJapproach breaks down. Around $\xi_{t p}$ we analyse (3.2) by zooming in around the turning point. After introducing $z=2 a^{-\frac{2}{3}}(1-x)$ into (3.2) we find the Airy equation

$$
W_{z z}-z W=0
$$


The solution of this equation is given by a linear combination of the Airy functions

$$
W(z)=C_{A i} \operatorname{Ai}(z)+C_{B i} \operatorname{Bi}(z)
$$

Now we match this solution to the left of $\xi_{t p}$ to the WKBJ-expression in (7.4). For that we introduce $\xi=\frac{2}{a}-y$, with $y>0$, $a^{-\frac{1}{3}} \ll y \ll \frac{1}{a}$. In terms of $y$, we find $\frac{2}{a} F\left(\frac{a}{2} \xi\right)=-\frac{2}{3} a^{\frac{1}{2}} y^{\frac{3}{2}}$ such that the WKBJ-solution (7.2) is to leading order given by

$$
W(y)=a^{-\frac{1}{4}} y^{-\frac{1}{4}}\left[C_{-} e^{\frac{2}{3} a^{\frac{1}{2}} y^{\frac{3}{2}}}+C_{+} e^{-\frac{2}{3} a^{\frac{1}{2}} y^{\frac{3}{2}}}\right] .
$$

Using the asymptotic expressions for $y \gg 1$ as known for the Airy solutions, we find that this WKBJ-solution can be matched to the Airy solution (7.7) upon choosing

$$
C_{+}=\frac{1}{2} a^{\frac{1}{6}} \pi^{-\frac{1}{2}} C_{A i}, \quad C_{-}=a^{\frac{1}{6}} \pi^{-\frac{1}{2}} C_{B i}
$$

In a similar way as in $[5,13]$, the matching can be continued to the right-hand side of the turning point and further into the far field. This analysis is given in detail in [13], therefore we just give the results here and refer to this analysis. It is found that the constants in the solutions are related as

$$
\begin{aligned}
C_{A i} & =i C_{B i} \\
\mu & =\sqrt{2} \pi^{-\frac{1}{2}} a^{\frac{d-3}{2}+\frac{1}{6}} e^{\frac{i}{a} \log a+i \frac{\pi}{4}} C_{B i}
\end{aligned}
$$

Finally, combining this with the above expression for $C_{-}$in (7.6) leads to

$$
\mu=2 a^{\frac{d-3}{2}} e^{\frac{i}{a} \log a+i \frac{\pi}{4}} \delta^{\frac{1-d}{2}} e^{\frac{1}{\delta}-\frac{\pi}{2 a}}
$$

completing the matching between the bump solution and the far field solution.

\section{Evaluating the integral expression}

In this section we evaluate the integral expression (2.7), as given in Lemma 2.1, to obtain a relation between the parameters in the problem. The relation that we find corresponds, to leading order, to the expression for $K_{0},(5.13)$, as found in Section 5.1.

We determine the integral expression (2.7) for $\xi \gg \frac{1}{a}$. Using the decomposition of $Q$ in amplitude and phase as given in (2.6), leads for $\xi \gg \frac{1}{a}$ to the following expression for $A$ and $\psi$

$$
A=\frac{|\mu|}{\xi}\left(1+\mathcal{O}\left(\frac{1}{a \xi^{2}}\right)\right) \quad \text { and } \quad \psi=-\frac{1}{a \xi}\left(1+\mathcal{O}\left(\frac{1}{a \xi^{3}}\right)\right) .
$$

Substituting these asymptotic estimates into expression (2.7), it follows that for $\xi \gg \frac{1}{a}$

$$
\frac{a \xi}{2}=\frac{\xi(2-d)}{|\mu|^{2}} \int_{0}^{\xi} A^{2} \psi d x+\frac{\xi \varepsilon}{|\mu|^{2}} \int_{0}^{\xi} x A\left(A_{x x}-A \psi^{2}+\frac{d-1}{x} A_{x}-b A^{3}\right) d x
$$


where both integrals converge as $\xi \rightarrow \infty$. Thus, letting $\xi \rightarrow \infty$, we find the exact expression,

$$
|\mu|^{2}=\frac{2(2-d)}{a} \int_{0}^{\infty} A^{2} \psi d x+\frac{2 \varepsilon}{a} \int_{0}^{\infty} x A\left(A_{x x}-A \psi^{2}+\frac{d-1}{x} A_{x}-b A^{3}\right) d x .
$$

To obtain the resulting parameter branch (2.2) as given in Section 2, we use the relation (7.8) that was determined for $\mu$ in the matching analysis in Section 7. A second estimate for $\mu$ follows from (global) estimates of both of the integral terms in $(8.3)$.

In order to obtain the expression for the two integrals in expression (8.3) over the whole (positive) real line, we determine the integrals in different regions as distinguished in the matching analysis. For this we use approximations for the amplitude $A$ and $\psi$ as obtained in Sections 3-7. It turns out that the main contribution to both integrals comes from the bump region; the other regions yield contributions that are higher order compared to the one in the bump region.

In constructing the solution, we had to study the solution in the WKBJ-region in Section 7. This region has a width of $\mathcal{O}\left(\frac{1}{a}\right)$, and hence we do need to determine the integral over this region as well. This is different from the analysis in [5]; there this was not necessary.

In the subsequent sections, we study the regions separately. We start every section by stating the amplitude $A$ and gradient of the phase $\psi$ as found for the solution in previous sections. Using these expressions, we obtain the integrals.

Note that all the analysis is to leading order, most of the time, we will refrain from mentioning this. Moreover, we use in the following analysis that $\kappa=1$ to leading order, although we only use this in the last step of each evaluation.

\subsection{The integral expression in the bump region}

In this section, we integrate over the bump region where the bump is found at $\xi=\frac{\kappa}{\delta}$. Hence, we integrate from $\xi=-z_{1}+\frac{\kappa}{\delta}$ up to $\xi=z_{2}+\frac{\kappa}{\delta}$, where $z_{1}, z_{2}>0, z_{1}, z_{2} \gg 1$, $z_{2} \ll \frac{1}{\delta}$ and $z_{1} \ll \log \frac{1}{\delta}$. In this region, we determine from Section 5 that

$$
\begin{aligned}
& \psi=-\frac{a \kappa}{2 \delta} \\
& A=\sqrt{2} \operatorname{sech}\left(\xi-\frac{\kappa}{\delta}\right) .
\end{aligned}
$$

The first integral in expression (8.3) is then given by

$$
\begin{aligned}
\int_{-z_{1}+\frac{\kappa}{\delta}}^{z_{2}+\frac{\kappa}{\delta}} A^{2} \psi d x & =\int_{-z_{1}}^{z_{2}}-\frac{a \kappa}{\delta} \operatorname{sech}^{2}(s) d s \\
& =-\frac{2 a \kappa}{\delta}=-\frac{2 a}{\delta}
\end{aligned}
$$

to leading order. 
The second term in expression (8.3) can be evaluated as

$$
\begin{aligned}
& \int_{-z_{1}+\frac{\kappa}{\delta}}^{z_{2}+\frac{\kappa}{\delta}} x A\left(A_{x x}-A \psi^{2}+\frac{d-1}{x} A_{x}-b A^{3}\right) d x \\
& =\int_{-z_{1}}^{z_{2}} 2 \frac{\kappa}{\delta}\left(\operatorname{sech}(s) \frac{d^{2}}{d s^{2}}(\operatorname{sech}(s))-\frac{a^{2} \kappa^{2}}{4 \delta^{2}} \operatorname{sech}^{2}(s)-2 b \operatorname{sech}^{4}(s)\right) d s \\
& \quad+\left[(d-1) \operatorname{sech}^{2}(s)\right]_{-z_{1}}^{z_{2}} \\
& =-\frac{4}{3 \delta}(1+4 b) .
\end{aligned}
$$

\subsection{The integral expression in the inner region}

In the inner region, we integrate from $\xi=0$ up to $\frac{\kappa}{\delta}-z_{1}$, where $z_{1}>0$ and $1 \ll z_{1} \ll \log \frac{1}{\delta}$ and we find in Section 4 that

$$
\begin{aligned}
& \psi=-\frac{a \xi}{2}, \\
& A=\alpha_{i n} e^{-\xi} M\left(\frac{d-1}{2}, d-1,2 \xi\right) .
\end{aligned}
$$

We evaluate most of the integrals in expression (8.3) by using the fact that the integral can be estimated by its value at the tail; for $\xi$ large. Hence, we use the asymptotic expansion for the Kummer function for $\xi \gg 1$, see [1],

$$
M\left(a_{1}, b_{1}, 2 \xi\right)=\frac{\Gamma\left(b_{1}\right)}{\Gamma\left(a_{1}\right)}(2 \xi)^{a_{1}-b_{1}} e^{2 \xi}+\text { hot }=\frac{2^{\frac{d-3}{2}}}{\sqrt{\pi}} \Gamma\left(\frac{d}{2}\right) \xi^{\frac{1-d}{2}} e^{2 \xi}+\text { hot, }
$$

and replace the lower bound $\xi=0$ of the integration by some $\xi=y_{3}$ where $1 \ll y_{3} \ll$ $\frac{\kappa}{\delta}-z_{1}$. This indeed gives the leading order of the integrals since $A$ is increasing and remains bounded for $\xi<y_{3}$.

The first integral in expression (8.3) is then given by

$$
\begin{aligned}
\mid \int_{0}^{\frac{\kappa}{\delta}-z_{1}} A^{2} \psi d x & =\left|-\frac{a}{2} \alpha_{i n}^{2} \int_{0}^{\frac{\kappa}{\delta}-z_{1}} x e^{-2 x}\left(M\left(\frac{d-1}{2}, d-1,2 x\right)\right)^{2} d x\right|, \\
& =\left|\frac{a}{\pi} 2^{d-4} \alpha_{i n}^{2}\left(\Gamma\left(\frac{d}{2}\right)\right)^{2} \int_{y_{3}}^{\frac{\kappa}{\delta}-z_{1}} x^{2-d} e^{2 x} d x\right|, \\
& =\left|a \alpha_{i n}^{2} \frac{2^{2 d-7}}{\pi}\left(\Gamma\left(\frac{d}{2}\right)\right)^{2}[\Gamma[3-d,-2 x]]_{y_{3}}^{\frac{\kappa}{\delta}-z_{1}}\right|, \\
& =\left|a \alpha_{i n}^{2} \frac{2^{d-5}}{\pi}\left(\Gamma\left(\frac{d}{2}\right)\right)^{2} \delta^{d-2} e^{2\left(\frac{1}{\delta}-z_{1}\right)}\right|, \\
& =\frac{a}{2 \delta} e^{-2 z_{1}} \ll \frac{a}{\delta}
\end{aligned}
$$

to leading order, and hence its contribution to the integral is smaller than that of the solution in the bump region (which is of $\mathcal{O}\left(\frac{1}{a}\right)$ ). 
The second integral in expression (8.3) is in this region also smaller than the contribution to the integral of the bump solution. We show this in Appendix A.

\subsection{The integral expression in the WKBJ-region}

In the construction of the solution, the WKBJ-region runs from $\xi_{1}=\frac{\kappa}{\delta}+z_{2}$ to $\xi_{2}=\frac{2}{a}-y_{2}$, where $z_{2}, y_{2}>0,1 \ll z_{2} \ll \frac{1}{\delta}$ and $a^{-\frac{1}{3}} \ll y_{2} \ll \frac{1}{a}$. Hence, this region has the size of order $\mathcal{O}\left(\frac{1}{a}\right)$, therefore we do need to determine the contribution of this region to the integral expression. Again, it will turn out that its contribution is much smaller than that of the bump region.

In the WKBJ-region, we find from (7.4) and the relation $C_{+}=\frac{1}{2} i C_{-}$that to leading order

$$
\begin{aligned}
\psi & =-\frac{a \xi}{2} \\
A^{2} & =\left|C_{-}\right|^{2} \xi^{1-d}\left(1-\frac{a^{2} \xi^{2}}{4}\right)^{-\frac{1}{2}}\left\{e^{-\frac{4}{a} F\left(\frac{a \xi}{2}\right)}+\frac{1}{4} e^{\frac{4}{a} F\left(\frac{a \xi}{2}\right)}\right\} \\
& =\left|C_{-}\right|^{2} \xi^{1-d}\left(1-\frac{a^{2} \xi^{2}}{4}\right)^{-\frac{1}{2}} e^{-\frac{4}{a} F\left(\frac{a \xi}{2}\right)}
\end{aligned}
$$

where $F$ is given by (7.3) and $C_{-}$by (7.6), see Section 7 . Here the second equality in the expression for $A^{2}$ is obtained by using that

$$
\begin{aligned}
0 & >-\frac{1}{3}\left(a y_{2}\right)^{\frac{3}{2}}=F\left(\frac{a \xi_{2}}{2}\right)>F\left(\frac{a \xi}{2}\right)>F\left(\frac{a \xi_{1}}{2}\right) \\
& =\frac{a}{2}\left(\frac{\kappa}{\delta}+z_{2}\right)-\frac{\pi}{4}, \text { and hence, } F\left(\frac{a \xi}{2}\right)<0 \text { and } \frac{1}{a}\left|F\left(\frac{a \xi}{2}\right)\right| \gg 1
\end{aligned}
$$

From this we conclude that the second term in $A^{2}$ is exponentially small, and hence higher order.

The first integral in expression (8.3) is then given by

$$
\begin{aligned}
\left|\int_{\xi_{1}}^{\xi_{2}} A^{2} \psi d x\right| & =\int_{\xi_{1}}^{\xi_{2}} \frac{1}{2} a\left|C_{-}\right|^{2} x^{2-d}\left(1-\frac{a^{2} x^{2}}{4}\right)^{-\frac{1}{2}} e^{-\frac{4}{a} F\left(\frac{a x}{2}\right)} d x, \\
& =d e \int_{\frac{a \xi_{1}}{2}}^{\frac{a \xi_{2}}{2}}\left(\frac{2}{a}\right)^{2-d}\left|C_{-}\right|^{2} s^{2-d}\left(1-s^{2}\right)^{-\frac{1}{2}} e^{-\frac{4}{a} F(s)} d s, \\
& \leqslant \frac{1}{2}\left(\frac{2}{a}\right)^{1-d}\left|C_{-}\right|^{2} \max _{s \in\left[\frac{a \xi_{1}}{2}, \frac{a \xi_{2}}{2}\right]}\left(s^{2-d}\left(1-s^{2}\right)^{-1}\right)\left[-e^{-\frac{4}{a} F(s)}\right]_{\frac{a \xi_{1}}{2}}^{\frac{a \xi_{2}}{2}}, \\
& =\frac{1}{2}\left(\frac{2}{a}\right)^{1-d}\left|C_{-}\right|^{2} \max _{2}\left\{\left(a y_{2}\right)^{-1},\left(\frac{a}{2 \delta}\right)^{2-d}\right\}\left\{-e^{-\frac{4}{a} F\left(\frac{a \xi_{2}}{2}\right)}+e^{-\frac{4}{a} F\left(\frac{a \xi_{1}}{2}\right)}\right\}, \\
& =\max \left\{2^{-d} a^{d-2} \delta^{1-d} y_{2}^{-1}, \frac{a}{2 \delta}\right\} e^{-2 z_{2}} \ll \frac{a}{\delta}
\end{aligned}
$$


to leading order. Again, the contribution of the above integral is smaller than the one in the bump region.

The second integral in expression (8.3) is determined in Appendix B.

\subsection{The integrals in the far field}

For the far field, we integrate over all $\xi \geqslant \frac{2}{a}+\tilde{y}$, where $\tilde{y}>0, \tilde{y} \gg \frac{1}{a}$. Then, we have

$$
\begin{aligned}
& \psi=-\frac{1}{a \xi}, \\
& A=|\mu| \xi^{-1} .
\end{aligned}
$$

Using this, the first integral in (8.3) is given by

$$
\begin{aligned}
\int_{\frac{2}{a}+\tilde{y}}^{\infty} A^{2} \psi d x & =-\frac{|\mu|^{2}}{a} \int_{\frac{2}{a}+\tilde{y}}^{\infty} x^{-3} d x \\
& =\frac{|\mu|^{2}}{2} a\left(\frac{2}{a}+\tilde{y}\right)^{-2}=\frac{|\mu|^{2}}{2} a \tilde{y}^{-2} \ll a^{3}|\mu|^{2} .
\end{aligned}
$$

Expression (7.8) found in Section 7.1 implies that $|\mu|$ is exponentially small, and therefore the contribution of the far field to the first integral is certainly smaller than the one in the bump region.

The second integral in (8.3) is obtained as

$$
\begin{aligned}
\int_{\frac{2}{a}+\tilde{y}}^{\infty} x A\left(A_{x x}-A \psi^{2}+\frac{d-1}{x} A_{x}-b A^{3}\right) d x & =|\mu|^{2} \int_{\frac{2}{a}+\tilde{y}}^{\infty}\left(-\frac{1}{a^{2}}-d+3-b|\mu|^{2}\right) x^{-3} d x \\
& =|\mu|^{2} \frac{1}{2}\left(-\frac{1}{a^{2}}-d+3-b|\mu|^{2}\right)\left(\frac{2}{a}+\tilde{y}\right)^{-2} \\
& \ll|\mu|^{2} \frac{1}{2}\left(3-\frac{1}{a^{2}}-d-b|\mu|^{2}\right) \tilde{y}^{-2} \ll|\mu|^{2}
\end{aligned}
$$

which again is an exponentially small contribution to the integral.

\section{The resulting relation for the parameters}

In this section, we collect all the results from the sections above and substitute these into the integral expression (8.3). This yields the following leading order relation between the parameters

$$
|\mu|^{2}=\frac{4(d-2)}{\delta}-\frac{8 \varepsilon}{3 a \delta}(1+4 b) .
$$

Note that the only contribution to right-hand side comes from the bump region. 
Now we use expression (7.8) for $\mu$, this gives

$$
4 a^{d-3} \delta^{2-d} e^{\frac{2}{\delta}-\frac{\pi}{a}}=d-2-\frac{2 \varepsilon}{3 a}(1+4 b)
$$

which, after setting $\delta=\log \frac{1}{a}$, results in relation (2.2) as given in the main result in Section 2.

The relation consists of a balance between an exponentially small term on the left-hand side and an algebraically small term on the right-hand side. The leading order of this relation is given by the right-hand side and yields

$$
\varepsilon=a \frac{3(d-2)}{2(1+4 b)} .
$$

This corresponds to relation (5.13) that was found for $K_{0}$ in the bump region analysis, in Section 5 , by using

$$
\varepsilon=K a \text { with } K=K_{0}+\text { hot. }
$$

\section{Discussion}

In this paper, we construct, by using asymptotic analysis, solutions to equation (1.3) for $a \ll 1$, which correspond to blowup solutions of the GL (1.1). These solutions have a maximum at $\xi_{\text {bump }}=\frac{\kappa}{\delta}$ where $\delta=\left(\log \frac{1}{a}\right)^{-1}$ and $Q(0)$ is algebraically small in $a$, see Section 2 for the statement of the main result. These solutions are essentially different from the solutions that were constructed in [5]. To compare, these solutions of [5] have a maximum at $\xi=\frac{\tilde{\kappa}}{a}$ and $Q(0)$ is exponentially small in $a$. However, the bifurcation curves on which both of the solutions are found are to leading order the same.

The solutions in [5] are found to be numerically stable under certain conditions whereas we expect the solutions found in this paper to be unstable. They have so far not been found in the numerical simulations in [5] and [10], nor in the numerical simulations of the NLS in [4] (which corresponds to the choice $\varepsilon=0$ and $d$ exponentially close to $d=2$ in (1.1)). In order to study the stability of the solutions analytically, a similar analytic approach as in [13] could be performed; there the stability of solutions with $Q(0)$ is studied by using Evans function techniques.

\section{Acknowledgements}

The work of V.R. was partially supported by a VIDI-grant of the Dutch Science Organisation (NWO).

\section{Appendix A The second term of (8.3) in the inner region}

In this appendix, we determine the contribution of the solution in the inner region to the second integral in the integral expression (8.3). Using the expressions for $\psi(8.4)$ and $A$ 
(8.5) and that

$$
\begin{aligned}
A_{x} & =\alpha_{i n} e^{-\xi}\left[M\left(\frac{d+1}{2}, d, 2 \xi\right)-M\left(\frac{d-1}{2}, d-1,2 \xi\right)\right] \\
& =\frac{2^{\frac{d-3}{2}} \alpha_{i n}(d-1)}{\sqrt{\pi}(d+1)} \Gamma\left(\frac{d}{2}\right) \xi^{\frac{1-d}{2}} e^{\xi}+\text { hot }
\end{aligned}
$$

we find

$$
\begin{aligned}
&\left|\int_{0}^{\frac{\kappa}{\delta}-z_{1}} x A\left(A_{x x}-A \psi^{2}+\frac{d-1}{x} A_{x}-b A^{3}\right) d x\right| \\
&=\left|\left[x A A_{x}+\frac{1}{2}(d-2) A^{2}\right]_{0}^{\frac{\kappa}{\delta}-z_{1}}-\int_{0}^{\frac{\kappa}{\delta}-z_{1}} x A_{x}^{2}+x A^{2}\left(\psi^{2}+b A^{2}\right) d x\right| \\
&=\frac{2^{d-3} \alpha_{i n}^{2}}{\pi}\left(\Gamma\left(\frac{d}{2}\right)\right)^{2} \mid\left[x^{1-d} e^{2 x}\left(\frac{d-1}{d+1} x+\frac{d-2}{2}\right)\right]_{0}^{\frac{\kappa}{\delta}-z_{1}} \\
&-\int_{y_{3}}^{\frac{\kappa}{\delta}-z_{1}}\left(\frac{d-1}{d+1}\right)^{2} x^{2-d} e^{2 x}+\frac{a^{2}}{4} x^{4-d} e^{2 x}+b \frac{2^{d-3} \alpha_{i n}^{2}}{\pi}\left(\Gamma\left(\frac{d}{2}\right)\right)^{2} x^{3-2 d} e^{4 x} d x \mid \\
&= 2 \delta^{1-d} e^{-\frac{2}{\delta}} \mid \frac{d-1}{d+1}\left(\frac{\kappa}{\delta}-z_{1}\right)^{2-d} e^{2\left(\frac{\kappa}{\delta}-z_{1}\right)} \\
&-\left[\left(\frac{d-1}{d+1}\right)^{2}(-1)^{d} 2^{d-3} \Gamma[3-d,-2 x]+a^{2}(-1)^{d} 2^{d-7} \Gamma[5-d,-2 x]\right. \\
&\left.-\delta^{1-d} e^{-\frac{2}{\delta}} b 2^{4 d-7}(-1)^{2 d} \Gamma[4-2 d,-4 x]\right]_{y_{3}}^{\frac{\kappa}{\delta}-z_{1}} \mid \\
&= \mid 2 \frac{d-1}{d+1} \delta^{-1} e^{-2 z_{1}}-2 \delta^{1-d} e^{-\frac{2}{\delta}}\left[\left(\frac{d-1}{d+1}\right)^{2} \frac{1}{2} x^{2-d} e^{2 x}\right. \\
&= \frac{1}{\delta}|b| e^{-1} e^{-2 z_{1}} \frac{(d-1)(d+3)}{(d+1)^{2}}-b e^{-4 z_{1}} \delta^{-1} \mid \\
&-\frac{a^{2}}{8} x^{4-d} e^{2 x}+\left.\frac{1}{2} \delta^{1-d} e^{-\frac{2}{\delta}} b x^{3-2 d} e^{4 x}\right|_{y_{3}} ^{\frac{\kappa}{\delta}-z_{1}} \mid
\end{aligned}
$$

Again, this is indeed smaller than the contribution of the bump region to integral.

\section{Appendix B The second term of (8.3) in the WKBJ-region}

In the WKBJ-region, the second integral in (8.3) is evaluated using that

$$
\begin{aligned}
& \left|\int_{\xi_{1}}^{\xi_{2}} x A\left(A_{x x}-A \psi^{2}+\frac{d-1}{x} A_{x}-b A^{3}\right) d x\right| \\
& \quad=\left|\left[x A A_{x}+\frac{1}{2}(d-2) A^{2}\right]_{\xi_{1}}^{\xi_{2}}-\int_{\xi_{1}}^{\xi_{2}} x A_{x}^{2}+x A^{2}\left(\psi^{2}+b A^{2}\right) d x\right| .
\end{aligned}
$$


Now we evaluate both of these terms separately using the expressions for $\psi(8.6)$ and $A$ (8.7). This second expression also gives

$$
\begin{aligned}
A_{\xi} & =\frac{1-d}{2} \xi^{-1} A+\frac{a^{2} \xi}{8}\left(1-\frac{a^{2} \xi^{2}}{4}\right)^{-1} A-\left|C_{-}\right| \xi^{\frac{1-d}{2}}\left(1-\frac{a^{2} \xi^{2}}{4}\right)^{-\frac{1}{4}} e^{-\frac{2}{a} F\left(\frac{a \xi}{2}\right)} F_{\xi}\left(\frac{a \xi}{2}\right) \\
& =A\left[\frac{1}{2} \xi^{-1}\left(1-\frac{a^{2} \xi^{2}}{4}\right)^{-1}\left(1-d+d \frac{a^{2} \xi^{2}}{4}\right)-\left(1-\frac{a^{2} \xi^{2}}{4}\right)^{-\frac{1}{2}}\right] .
\end{aligned}
$$

First, we determine

$$
\begin{aligned}
{\left[x A A_{x}+\frac{1}{2}(d-2) A^{2}\right]_{\xi_{1}}^{\xi_{2}}=} & {\left[A ^ { 2 } \left\{\frac{1}{2}\left(1-d+d \frac{a^{2} x^{2}}{4}\right)\left(1-\frac{a^{2} x^{2}}{4}\right)^{-1}\right.\right.} \\
& \left.\left.+\frac{d-2}{2}-x\left(1-\frac{a^{2} x^{2}}{4}\right)^{-\frac{1}{2}}\right\}\right]_{\xi_{1}}^{\xi_{2}} \\
= & {\left[\frac{1}{4} x^{1-d}\left(1-\frac{a^{2} x^{2}}{4}\right)^{-\frac{3}{2}}\left|C_{-}\right|^{2} e^{-\frac{4}{a} F\left(\frac{a x}{2}\right)}\left(a^{2} x^{2}-2\right)\right.} \\
= & \frac{1}{2} \xi_{1}^{1-d}\left|C_{-}\right|^{2} e^{-\frac{4}{a} F\left(\frac{a}{2} \xi_{1}\right)}\left(8 \xi_{1}-1\right) \\
= & \frac{4}{\delta} e^{-2 z_{2}} \ll \frac{1}{\delta} .
\end{aligned}
$$

Then,

$$
\begin{aligned}
\left|\int_{\xi_{1}}^{\xi_{2}} x A^{2} \psi^{2} d x\right| & =\left|-\frac{a}{2} \int_{\xi_{1}}^{\xi_{2}} x^{2} A^{2} \psi d x\right| \\
& \leqslant \frac{a}{2} \xi_{2}^{2}\left|\int_{\xi_{1}}^{\xi_{2}} A^{2} \psi d x\right|=\frac{2}{a} \int_{\xi_{1}}^{\xi_{2}} A^{2} \psi d x \ll \frac{2}{\delta},
\end{aligned}
$$

using the estimate found in (8.8).

Also, we estimate

$$
\begin{aligned}
\left|\int_{\xi_{1}}^{\xi_{2}} x A_{x}^{2} d x\right| & =\mid \int_{\xi_{1}}^{\xi_{2}} A^{2}\left\{\frac{1}{4} x^{-1}\left(1-\frac{a^{2} x^{2}}{4}\right)^{-2}\left(1-d\left(1-\frac{a^{2} x^{2}}{4}\right)\right)^{2}\right. \\
& \left.-\left(1-\frac{a^{2} x^{2}}{4}\right)^{-\frac{1}{2}}\left(1-d\left(1-\frac{a^{2} x^{2}}{4}\right)\right)+8 x\left(1-\frac{a^{2} x^{2}}{4}\right)\right\} d x \mid
\end{aligned}
$$




$$
\begin{aligned}
= & \left|C_{-}\right|^{2} \mid \int_{\xi_{1}}^{\xi_{2}}\left\{\frac{1}{2} x^{-d}\left(1-\frac{a^{2} x^{2}}{4}\right)^{-\frac{5}{2}}\left(1-d\left(1-\frac{a^{2} x^{2}}{4}\right)\right)^{2}\right. \\
& \left.-x^{1-d}\left(1-\frac{a^{2} x^{2}}{4}\right)^{-1}\left(1-d\left(1-\frac{a^{2} x^{2}}{4}\right)\right)+x^{2-d}\left(1-\frac{a^{2} x^{2}}{4}\right)^{\frac{1}{2}}\right\} e^{-\frac{4}{a} F\left(\frac{a x}{2}\right)} d x \mid \\
\leqslant & \left|C_{-}\right|^{2}\left(\frac{2}{a}\right)^{-d}\left[e^{-\frac{4}{a} F(x)}\right]_{\frac{a \xi_{1}}{2}}^{\frac{a \xi_{2}}{2}}\left\{\frac{1}{4} \max _{s \in\left[\frac{a \xi_{1}}{2}, \frac{a \xi_{2}}{2}\right]}\left(s^{-d}\left(1-s^{2}\right)^{-3}\left(1-d\left(1-s^{2}\right)\right)^{2}\right)\right. \\
& \left.-\frac{1}{a} \max _{s \in\left[\frac{a \xi_{1}}{2}, \frac{a \xi_{2}}{2}\right]}\left(s^{1-d}\left(1-s^{2}\right)^{-\frac{3}{2}}\left(1-d\left(1-s^{2}\right)\right)\right) \frac{2}{a^{2}} \max _{s \in\left[\frac{a \xi_{1}}{2}, \frac{a_{2}}{2}\right]}\left(s^{2-d}\right)\right\} \\
\leqslant & 8 \delta^{-1} e^{-2 z_{2}} \ll \delta^{-1} .
\end{aligned}
$$

Finally,

$$
\begin{aligned}
\int_{\xi_{1}}^{\xi_{2}} x A^{4} d x & =\left|C_{-}\right|^{4} \int_{\xi_{1}}^{\xi_{2}} x^{3-2 d}\left(1-\frac{a^{2} x^{2}}{4}\right)^{-1} e^{-\frac{8}{a} F\left(\frac{a x}{2}\right)} d x \\
& =\left|C_{-}\right|^{4} \int_{\frac{a \xi_{1}}{2}}^{\frac{a \xi_{2}}{2}}\left(\frac{2}{a}\right)^{4-2 d} s^{3-2 d}\left(1-s^{2}\right)^{-1} e^{-\frac{8}{a} F(s)} d s \\
& =\frac{1}{4}\left|C_{-}\right|^{4}\left(\frac{2}{a}\right)^{3-2 d}\left[-e^{-\frac{8}{a} F(s)}\right]_{\frac{a \xi_{1}}{2}}^{\frac{a \xi_{2}}{2}} \max \\
& \left.=2^{3-2 d} \frac{1}{\delta} e^{-4 z_{2}}+\operatorname{hot} \ll \frac{1}{\delta}, \frac{a \xi_{2}}{2}\right]
\end{aligned}
$$

Hence, taking these terms together, the contribution of the WKBJ-region to the second integral in expression (8.3) is smaller than that in the bump region.

\section{References}

[1] Abramovitz, M. \& Stegun, I. A. (1972) Handbook of Mathematical Functions with Formulas, Graphs and Mathematical Tables, Dover, New York.

[2] Aranson, I. S. \& Kramer, L. (2002) The world of the complex Ginzburg-Landau equation. Rev. Mod. Phys. 74, 99-143.

[3] Brand, H. R., Lomdahl, P. S. \& Newell, A. C. (1986) Benjamir-Feir turbulence in convective binary fluid mixtures. Physica D 23, 345-362.

[4] Budd, C. J. (2002) Asymptotics of multi-bump blow-up self-similar solutions of the nonlinear Schrödinger equation. SIAM J. Appl. Math. 62(3), 801-830.

[5] Budd, C. J., RotTschäFer, V. \& Williams, J. F. (2005) Multi-bump, blow-up, self-similar solutions of the complex Ginzburg-Landau equation. SIAM J. Appl. Dyn. Sys. 4(3), 649-678.

[6] Davey, A., Hocking, L. M. \& Stewartson, K. (1974) On the nonlinear evolution of threedimensional disturbances in plane Poiseuille flow. J. Fluid Mech. 63, 529-536.

[7] DiPrima, R. C. \& Swinney, H. L. (1981) Instabilities and transition in flow between concentric cylinders in hydrodynamic instabilities and the transition to turbulence. In: H. Swinney \& J. Gollub (editors), Topics in Applied Physics, Vol. 45, Springer-Verlag, New York.

[8] Mielke, A. (2002) The Ginzburg-Landau equation in its role as a modulation equation. In: B. Fiedler (editor), Handbook of Dynamical Systems, Vol. 2, North-Holland, Amsterdam, Netherlands, pp. 759-834. 
[9] Newell, A. C. \& Whitehead, J. A. (1969) Finite bandwidth, finite amplitude convection. J. Fluid Mech. 28, 279-303.

[10] Plecháč, P. \& Šverák, V. (2001) On self-similar singular solutions of the complex GinzburgLandau equation. Comm. Pure Appl. Math. 54, 1215-1242.

[11] RotTsChäFER, V. (2008) Multi-bump, self-similar, blowup solutions of the Ginzburg-Landau equation. Physica D 237, 510-539.

[12] RotTSCHÄFER, V. \& KAPER, T. (2003) Geometric theory for multi-bump, self-similar, blowup solutions of the cubic nonlinear Schrödinger equation. Nonlinearity 16(3), 929-961.

[13] Schans, M. v.D. (In preparation) PhD thesis. Leiden University, Leiden, Netherlands.

[14] Schans, M. V.D., Doelman, A. \& V. Rottschäfer (In preparation) Stability of ring-type blowup solutions of the Ginzburg-Landau equation.

[15] Stewartson, K. \& Stuart, J. (1971) A nonlinear instability theory for a wave system in plane Poiseuille flow. J. Fluid Mech. 48, 529-545.

[16] Sulem, C. \& Sulem, P. L. (1999) The Nonlinear Schrödinger Equation: Self-Focusing and Wave Collapse, Applied Mathematical Sciences Series 139, Springer, New York. 